\title{
Cupping for Treating Neck Pain in Video Display Terminal (VDT) Users: A Randomized Controlled Pilot Trial
}

\author{
Tae-Hun $\mathrm{KIM}^{1}$, Jung Won KANG ${ }^{1,2}$, Kun Hyung $\mathrm{KIM}^{1,3}$, Min Hee LEE ${ }^{1}$, Jung Eun KIM ${ }^{1}$, \\ Joo-Hee KIM ${ }^{1}$, Seunghoon LEE ${ }^{1}$, Mi-Suk SHIN ${ }^{1}$, So-Young JUng ${ }^{1}$, Ae-Ran KIM ${ }^{1}$, \\ Hyo-Ju PARK ${ }^{1}$ and Kwon Eui Hong ${ }^{4}$
}

\begin{abstract}
${ }^{1}$ Korea Institute of Oriental Medicine, South Korea, ${ }^{2}$ Department of Acupuncture and Moxibustion, College of Oriental Medicine, Kyung-Hee University, South Korea, ${ }^{3}$ Department of Acupuncture and Moxibustion, Korean Medicine Hospital, Pusan National University, South Korea and ${ }^{4}$ Department of Acupuncture and Moxibustion, Daejeon University, South Korea
\end{abstract}

\begin{abstract}
Cupping for Treating Neck Pain in Video Display Terminal (VDT) Users: A Randomized Controlled Pilot Trial: Tae-Hun KıM, et al. Korea Institute of Oriental Medicine, South Korea-Objectives: This was a randomized controlled pilot trial to evaluate the effectiveness of cupping therapy for neck pain in video display terminal (VDT) workers. Methods: Forty VDT workers with moderate to severe neck pain were recruited from May, 2011 to February, 2012. Participants were randomly allocated into one of the two interventions: 6 sessions of wet and dry cupping or heating pad application. The participants were offered an exercise program to perform during the participation period. A 0 to 100 numeric rating scale (NRS) for neck pain, measure yourself medical outcome profile 2 score (MYMOP2 score), cervical spine range of motion (Cspine ROM), neck disability index (NDI), the EuroQol health index (EQ-5D), short form stress response inventory (SRI-SF) and fatigue severity scale (FSS) were assessed at several points during a 7 -week period. Results: Compared with a heating pad, cupping was more effective in improving pain (adjusted NRS difference: $-1.29[95 \% \mathrm{Cl}-1.61,-0.97]$ at 3 weeks $(p=0.025)$ and $-1.16[-1.48,-0.84]$ at 7 weeks $(p=0.005))$, neck function (adjusted NDI difference: -0.79 [-1.11, -0.47] at $3(p=0.0039)$ and 7 weeks $(p<0.0001))$ and discomfort (adjusted MYMOP2 difference score: $-0.72[-1.04$ to -0.40$]$ at 3 weeks and $-0.92[-1.24,-0.60]$ at 7 weeks). Significant improvement in EQ-5D was observed at 7 weeks $(1.0[0.88,1.0]$ with cupping and $0.91[0.86,0.91]$ with heating pad treatment, $p=0.0054)$. Four participants reported mild adverse events of
\end{abstract}

Received May 29, 2012; Accepted Jul 23, 2012

Published online in J-STAGE Sep 1, 2012

Correspondence to: T.-H. Kim, Korea Institute of Oriental Medicine, 488, Expo-ro, Yuseong, Daejeon 305-811, South Korea (e-mail: rockandmineral@gmail.com) cupping. Conclusion: Two weeks of cupping therapy and an exercise program may be effective in reducing pain and improving neck function in VDT workers.

(J Occup Health 2012; 54: 416-426)

Key words: Neck pain, Randomized controlled trial, Video display terminals (VDT), Video display terminals and cupping

Work involving video display terminals (VDTs) is very common in modern society. VDT work has accompanied the sharp increase in the use of personal computers for work in the last several decades. According to a national survey of the Japan Ministry of Health, Labour and Welfare, Japanese laborers who engaged in VDT-related work comprised only $15.3 \%$ of all the working population in 1998, but after 10 years, that fraction increased to $79.2 \%{ }^{1)}$. German census data from 2004 suggested that twenty-one million people, comprising $59 \%$ of all hired workers, were engaged in computer-related work during daily life ${ }^{2)}$.

Along with the rapid changes of working environments into VDT stations, various symptoms related to VDT use have been increasingly reported. Sixtythree per cent of office workers have been suggested to have musculoskeletal symptoms, and $42 \%$ of them might suffer head and neck pain ${ }^{3)}$. Thirtysix per cent of VDT workers have the symptoms of dry eye syndrome or were diagnosed with the prob$\mathrm{lem}^{4}$. In addition, eye symptoms such as asthenopia, pain and fatigue are suffered by many workers ${ }^{1,5)}$. Recent systematic reviews (SRs) suggest that there is evidence that tension neck syndromes of VDT workers are strongly related to the use of computers ${ }^{6}$. To prevent or treat these symptoms in VDT workers, 
the workstation guidelines for such tools as monitor displays, keyboards, tables, chairs and even working posture are currently available in Korea ${ }^{7}$.

Cupping is an intervention that has been widely used in Asia, the Middle East ${ }^{8)}$ and European countries $^{9)}$ as an alternative therapy. In Korea, most doctors who practice Korean medicine (about 90\%) are currently using cupping therapy in clinical practice $^{10,11)}$. In Korean medicine (KM), cupping has been employed for facilitating the circulation of qi and blood and removing blood stasis using cupping therapy devices through suction and negative pressure $^{12)}$. According to a recent survey of Korean medicinal doctors, cupping is primarily used for the treatment of musculoskeletal diseases (96\%), and the most frequently used points for cupping are the neck and shoulder $(94 \%)^{13)}$. Considering that a recent SR suggested that acupuncture, another traditional intervention that stimulates acupuncture points similar to cupping therapy, may possess short-term efficacy and effectiveness for the treatment of neck pain ${ }^{14)}$, clinical evidence concerning the effectiveness of cupping for neck pain also needs to be evaluated through rigorous clinical trials. For this purpose, we evaluated the feasibility of a large sample size clinical study and conducted a basic statistical analysis to evaluate the potential effectiveness and safety of cupping for neck pain through this randomized controlled pilot trial.

\section{Methods}

This was a randomized controlled pilot trial. The objective of this study was to compare the effects of cupping and heating pad therapies on neck pain, functional disability and quality of life in VDT workers and to evaluate the feasibility of this intervention for a large, randomized controlled trial as well.

Forty VDT workers with neck pain were recruited at the Oriental Medicine Hospital of Daejeon University, Daejeon City, South Korea, through advertisement in local newspapers and web pages of local companies and universities from May, 2011 to February, 2012. VDT users who worked with computers at least 20 hours a week and suffered work-related neck pain continuously for at least 3 months with an average severity over 40 on a 100 numeric rating scale (NRS) were included in the study. Participants who had serious conditions of the spine and spinal cord (e.g., ankylosing spondylitis), infectious disease, spinal fracture, myelopathy, acute herniation of the cervical intervertebral disk within 3 months, malignancy, immune disorders or whiplash injuries within the previous year were excluded. Cupping therapy involves an invasive procedure, so participants who had blood-borne diseases or hemostatic abnormalities, such as AIDS, active hepatitis, syphilis, hemophilia and anemia, or took anticoagulants or antiplatelet agents, such as warfarin, dipyridamole aspirin and ticlopidine, were excluded. Random sequences for group allocation of the participants were generated with a computer software package $\left(\mathrm{SAS}^{\circledR}\right.$ Version 9.1.3, SAS institute Inc., Cary, NC, USA). Random number tables and randomization block sizes were controlled by an independent statistical expert. Group allocation results were concealed by opaque, sealed envelopes with serial numbers, and the participants were assigned at the second visit by opening envelops in a sequential manner after their eligibility was assessed at the first visit. Written informed consent was obtained from each participant. The institutional review board of the Oriental Medicine Hospital of Daejeon University approved the study protocol (board approval number: djomc-59-1), and the study protocol was registered before the start of this trial at the Clinical Research Information Service (CRIS), which is the only primary registry in South Korea (registration number: KCT0000106).

\section{Interventions}

Participants in both the cupping and heating pad groups attended the assigned treatments 3 times per week during a total of 2 weeks, and the symptoms related to VDT work were evaluated for 7 weeks. For the cupping therapy, various sizes of sterile disposable cups (Seongho Trade \& Company, Korea) from $1.5 \mathrm{~cm}$ to $5 \mathrm{~cm}$ in diameter were used. Six to ten tender points of the posterior neck, upper trapezius and perispinal area of the neck and thoracic spine, such as GV14, GV16, GV15, GV12, GB20, GB21, LI17, SI11, SI12, SI13, SI14, SI15, BL10, BL11, BL12, BL13, BL14, B115, BL16, BL17, BL41, BL42, BL43, BL44 and EX-HN15, were selected for treatment. Dry cupping without puncture of the skin was applied mainly, but wet cupping was also applied for up to 2 points in the case of lesions with blood-stasis symptoms, severe tenderness and prominent warmness. According to the predefined clean technique procedure $^{15)}$, cupping cups were attached after 3 to 5 cycles of pumping for negative pressure by cupping pump and retained for 5 to 10 minutes according to the patient's general condition and symptom severity. For wet cupping, the skin was punctured 6 times to a 2-mm depth around the cupping sites with 26-gauge disposable lancets (Tianjin Haing Lim Sou Won Medical Instrument Co., China). At the sites where wet cupping was applied, 3 to $5 \mathrm{cc}$ of blood was drained. The selection of points and cupping methods were decided by practitioners after a brief consultation with the participants at each treatment session and was based on the basic guidelines of treatment from acupuncture textbooks and previous studies ${ }^{16,17)}$. 
Cupping treatment was conducted by qualified KM doctors who had over 4 years of practical clinical experience that had been acquired after finishing approximately 10 years of education and training (Appendix).

The participants assigned to the control group had heating pads warmed by hot water applied to the neck and upper trapezius for 10 minutes, 3 times per week for 2 weeks. Heating pad therapy is one of the most popular treatment tools for neck pain. It is used alone or concomitant with other physiotherapies for neck pain ${ }^{18)}$. The surface temperature of the heating pad was maintained at 55 degrees centigrade, and it was applied by independent physical therapists. A brochure explaining the relationship between VDT work and diseases, common treatments for patients with evidence of neck pain, advice on the life style modification when performing VDT work and exercise program details were offered to both treatment groups. During the 7-week participation period, neck and shoulder stretching exercises were performed in the office, and two types of deep cervical flexion exercises (i.e., craniocervical flexion and cervical flexion) were encouraged for home use ${ }^{19,20)}$. Except for this self-exercise program, all other neck pain treatments, such as acupuncture, yoga, Pilates, manipulation therapy, drug therapy, injection therapy and physical therapy were not allowed.

\section{Outcome assessments}

All the outcome assessment tools were developed in or translated into Korean and validated properly through previous validation studies. The primary outcome of this study was one-week average neck pain as measured by NRS (range 0 to 100) after the end of the 4-week treatment course. For evaluating the general improvement of pain, the average NRS of the last 7 days was assessed at every visit. In addition, a diary with the time series NRS data was collected from every participant at every treatment visit. The NRS scores before and after treatment in addition to after $1,4,8$ and 12 hours after treatment were assessed to test the short-term change of pain in both groups.

The secondary outcome variables were the measure yourself medical outcome profile 2 score (MYMOP2 score), cervical spine range of motion (C-spine ROM), neck disability index (NDI), EQ-5D, short form of stress response inventory (SRI-SF) and fatigue severity scale (FSS). MYMOP2 is a patient-generated instrument that is being used increasingly in primary care, particularly for assessing the effectiveness of complementary therapies ${ }^{21)}$. MYMOP2 is composed of 3 items: the first item is symptoms 1 and 2, which are the greatest and second-most physiological or psychological symptoms causing discomfort on a scale of 0 (the best) to 6 (the worst). The second item records how daily living activities are affected by symptoms 1 and 2 on a scale of 0 (the best) to 6 (the worst). The third item is a well-being score, recording on a scale from 0 (the best) to 6 (the worst). The MYMOP2 score is calculated by summing the symptom 1, symptom 2, activity and well-being scores and then dividing by the number of checked items. The score ranges from 0 (the best) to 6 (the worst). $\mathrm{C}$-spine ROM was evaluated before and after treatment and at the 4-week follow-up with CROM (cervical range of motion instrument, Performance Attainment Associates, Lindstrom, MN, USA). Cervical flexion, extension, left and right flexion and left and right rotation were assessed in an erect sitting posture in a straight-back chair. NDI is an inventory used to evaluate the function and disability of the neck. The instrument consists of 10 sections including pain intensity, personal care, lifting, reading, headaches, concentration, work, driving, sleeping and recreation $^{22)}$. All subsections are rated with a score from 0 (the best) to 5 (the worst), and the NDI score is presented as a percentage score, which is the total score*100/total possible score. The possible NDI scores range from $0 \%$ (the best) to $100 \%$ (the worst). The EuroQol health index (EQ-5D) is one of the most commonly used tools for evaluating current status of quality of life. The five dimensions of EQ-5D are mobility, self-care, usual activities, pain/discomfort and anxiety/depression. In this study, we adopted the EQ-5D-3L in which each domain has 3 levels, ranging from no problems to extreme problems. If each level is recorded as a number between 1 to 3 , and then each patient has a 5-digit number that ranges from 11,111 (the perfect health status) to 33,333 (the worst health status). The EQ-5D index of each patient was determined by using a transformed value from a 2007 study titled EQ-5D Korean Valuation Study Using Time Trade Off Method ${ }^{23)}$. The EQ-5D index values range from 1 for 11,111 to -0.171 for 33,333 . The SRI-SF is a tool for evaluating stress response during the preceding 7 days. It has 3 dimensions and a total of 22 questions with scores ranging from 1 (the best) to 5 (the worst). The SRI-SF score is the sum of the scores for all 22 questions, and it ranges from 22 (the best) to 110 (the worst) $^{24)}$. FSS is an inventory used to evaluate fatigue during the preceding 7 days. It has 9 items scored from 1 (the best) to 7 (the worst) individually. The FSS score is the sum of 9 item scores divided by 9 . It ranges from 1 (the best) to 7 (the worst ${ }^{25)}$. Adverse events (AEs), which were checked for by practitioners at every visit, were reported as AE type and frequency according to the criteria of the World Health Organisation (WHO Toxicity Grading 


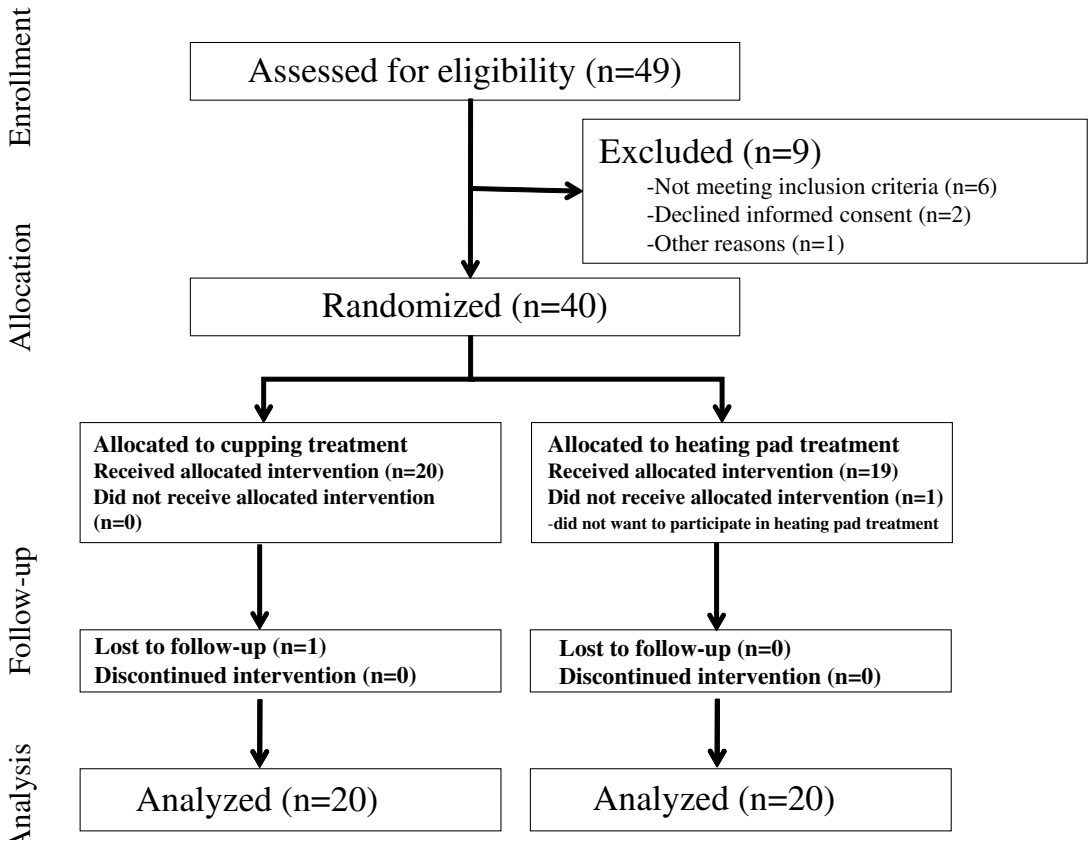

Fig. 1. CONSORT flow chart.

Scale for Determining The Severity of Adverse Events) ${ }^{26)}$.

\section{Statistical analysis and expectation assessment for} cupping therapy

This was a pilot trial, so sample size was not calculated at the beginning. Considering the feasibility for conducting a trial and the appropriate precision of this study result, we assigned a minimum of 20 participants to each group ${ }^{27)}$. Basically, an intentionto-treat analysis was conducted for statistical analysis, and missing data were amended with the last observation carried forward method. After applying a Shapiro-Wilk test for evaluating the normality of the data, a Chi-squared test or Fisher's exact test was applied to categorical data, and a two sample $t$-test or Wilcoxon rank-sum test was applied to continuous data for the statistical analysis of baseline characteristics between the two groups. The primary and secondary outcomes after treatment and at the 4-week follow-up were analyzed using analysis of covariance (ANCOVA). The baseline scores were used as covariates. The effect size for each outcome was calculated as eta-squared, and Cohen's criteria were used to interpret each effect ${ }^{28)}$. The time series data obtained from the NRS diaries were tested by repeated measures ANOVA for trend analysis. All statistical analyses were conducted using a computer software package (SAS ${ }^{\circledast}$ Version 9.1.3, SAS institute Inc., Cary, NC, USA). The expectation on the effectiveness of cupping was assessed in both of the groups before participation group allocation.

\section{Results}

In total, 49 participants were assessed for eligibility, and 40 participants were included in the study (Fig. 1). One participant was lost to follow-up from the cupping group, and one participant dropped out from the heating pad group because he did not want the heating pad treatment. The participants were mostly young adults and had about 2 years of neck pain history on average. Most of the participants used computers and/or watched TV over 30 hours per week. Along with neck pain, various VDT-related symptoms were observed in both groups. Asthenopia, back pain, shoulder and arm pain, fatigue and stressfulness were reported from over half of the participants (Table 1). During the work environment evaluation, most participants complained of problems with their workstation, such as uncomfortable chairs and keyboards or problems with sitting and typing postures (e.g., inappropriate angle of the wrist, knee and lumbar, Table 2). There were no significant differences between the two groups for all the baseline characteristics and expectations of the effectiveness of the cupping treatment (Tables 1 and 2).

\section{NRS}

One-week average neck pain was significantly improved in favor of cupping therapy after treatment and 4 weeks after the end of the whole treatment course. The eta-squared values were 0.1642 at 3 weeks and 0.1262 at 7 weeks. These observed values indicate a comparatively large effect of cupping 
Table 1. Demographic data in the cupping and heating pad groups

\begin{tabular}{|c|c|c|c|}
\hline Characteristics & $\begin{array}{l}\text { Cupping group } \\
\quad(n=20)\end{array}$ & $\begin{array}{l}\text { Heating pad group } \\
\qquad(\mathrm{n}=20)\end{array}$ & $p$-value \\
\hline Age, years (median [25\% to $75 \% \mathrm{IQR}])^{*}$ & $25.5[22.5$ to 40.5$]$ & 28 [25 to 41.5$]$ & 0.46 \\
\hline Sex M/F, No. ${ }^{\dagger}$ & $7 / 13$ & $11 / 9$ & 0.20 \\
\hline Duration of illness, years (median [25\% to $75 \% \mathrm{IQR}]$ )* & $2.5[1.85$ to 4$]$ & $2[1.25$ to 3.75$]$ & 0.50 \\
\hline Average time of computer or TV usage, $\mathrm{h} / \mathrm{wk}$ (median [25\% to $75 \% \mathrm{IQR}])^{*}$ & $32.5[28$ to 41$]$ & 31.25 [24 to 39.25$]$ & 0.39 \\
\hline Exercise $(\text { yes/no })^{\dagger}$ & $7 / 12$ & $8 / 12$ & 0.84 \\
\hline Past experience of drug therapy for neck pain (yes/no) & $1 / 19$ & $2 / 18$ & 1.0 \\
\hline Past experience of injection therapy for neck pain (yes/no) $)^{\ddagger}$ & $2 / 18$ & $2 / 18$ & 1.0 \\
\hline Past experience of physical therapy for neck pain (yes/no) ${ }^{\dagger}$ & $8 / 12$ & $10 / 10$ & 0.53 \\
\hline Past experience of cupping therapy for any reason (yes/no) ${ }^{\dagger}$ & $11 / 9$ & $15 / 5$ & 0.18 \\
\hline \multicolumn{4}{|l|}{ Concomitant symptoms related to VDT work } \\
\hline (1) Eye pain, No..$^{\dagger}$ & $4 / 16$ & $6 / 14$ & 0.47 \\
\hline (2) Dry eye, No. ${ }^{\dagger}$ & $8 / 12$ & $12 / 8$ & 0.21 \\
\hline (3) Asthenopia, No. ${ }^{\dagger}$ & $17 / 3$ & $16 / 4$ & 0.68 \\
\hline (4) Problems of visual acuity, No. ${ }^{\dagger}$ & $5 / 15$ & $10 / 10$ & 0.10 \\
\hline (5) Other ophthalmological problems, No..$^{\ddagger}$ & $2 / 18$ & $2 / 18$ & 1.0 \\
\hline (6) Headache, No. ${ }^{\dagger}$ & $5 / 15$ & $8 / 12$ & 0.31 \\
\hline (7) Back pain, No. ${ }^{\dagger}$ & $11 / 9$ & $12 / 8$ & 0.75 \\
\hline (8) Shoulder and arm pain, No. & $14 / 6$ & $17 / 3$ & 0.45 \\
\hline (9) Elbow pain, No. & $2 / 18$ & $4 / 16$ & 0.66 \\
\hline (10) Wrist pain, No. ${ }^{\dagger}$ & $8 / 12$ & $8 / 12$ & 1.0 \\
\hline (11) Finger pain, No. & $4 / 16$ & $2 / 18$ & 0.66 \\
\hline (12) Low back pain, No..$^{\dagger}$ & $8 / 12$ & $9 / 11$ & 0.75 \\
\hline (13) Other musculoskeletal pain, No. & $0 / 20$ & $0 / 20$ & 0 \\
\hline (14) Depressive mood, No. ${ }^{\ddagger}$ & $2 / 18$ & $4 / 16$ & 0.66 \\
\hline (15) Fatigue, No. ${ }^{\dagger}$ & $13 / 7$ & $15 / 5$ & 0.49 \\
\hline (16) Stressfulness, No. ${ }^{\dagger}$ & $10 / 10$ & $10 / 10$ & 1.0 \\
\hline (17) Other psychological symptoms, No." & $1 / 19$ & $0 / 20$ & 1.0 \\
\hline (18) Skin problems, No. & $4 / 16$ & $5 / 15$ & 1.0 \\
\hline (19) Dyspepsia, No. & $3 / 17$ & $2 / 18$ & 1.0 \\
\hline (20) Other symptoms, No. & $0 / 20$ & $1 / 19$ & 1.0 \\
\hline $\begin{array}{l}\text { Assessment of expectation on the effectiveness of cupping (median [25\% to } \\
75 \% \mathrm{IQR}] \text { )* }\end{array}$ & $7[5.5,7]$ & $7[6,8]$ & 0.38 \\
\hline
\end{tabular}

*The Wilcoxon rank-sum test was used for statistical analysis. ‘The Chi-squared test was used for statistical analysis. ${ }^{\ddagger}$ The Fisher’s exact test was used for statistical analysis. IQR: interquartile range.

on neck pain after 2 weeks of treatment and at the 4-week follow-up (Table 3).

Time series data from the NRS diaries suggest that cupping decreased neck pain immediately after the treatment, and this analgesic effect was maintained for at least 12 hours when compared with the heating pad treatment (Fig. 2). The repeated measures ANOVA results suggested that trends in the variation of pain severity in both groups moved in a similar pattern during the first 4 hours after treatment, but they were changed after 8 hours $(p=0.010$ at 8 hours and $p=0.009$ at 12 hours). These results indicated that the NRS for neck pain in the cupping group continued to decrease after 8 hours but did not for the heating pad group.

\section{MYMOP2}

Symptom 1 of MYMOP2 before treatment was neck pain alone (21 participants), shoulder pain alone (9), neck and shoulder pain (5), low-back pain (2), dizziness (1), fatigue (1) and stress (1). About half of the participants did not have a symptom 2 (18 participants), but neck pain (9), shoulder pain (5), headache (2), low-back pain (1), fatigue (1), elbow pain (1), upper-back pain (2) and wrist pain (1) were reported as symptom 2 in the other participants. The mean 
Table 2. Evaluation of the workstation in the cupping and heating pad groups

\begin{tabular}{|c|c|c|c|c|}
\hline \multicolumn{2}{|c|}{ Workstation evaluation [50] } & \multirow{2}{*}{$\begin{array}{c}\begin{array}{c}\text { Cupping group } \\
(\mathrm{n}=21)\end{array} \\
1 / 19\end{array}$} & \multirow{2}{*}{$\begin{array}{l}\text { Heating pad group } \\
(\mathrm{n}=20)\end{array}$} & \multirow{2}{*}{$\frac{p \text {-value }}{1.0}$} \\
\hline (1) & Keyboard is ergonomic? (yes/no)* & & & \\
\hline (2) & The mouse is held in a neutral position of the wrist? (yes/no)* & $15 / 5$ & $17 / 3$ & 0.69 \\
\hline (3) & Top of computer screen is at the eye level? (yes/no) ${ }^{\dagger}$ & $9 / 11$ & $15 / 5$ & 0.05 \\
\hline (4) & Chair height is appropriate? (yes/no)* & $16 / 4$ & $17 / 3$ & 1.0 \\
\hline (5) & There is enough space between the chair and calves? (yes/no)* & $17 / 3$ & $17 / 3$ & 1.0 \\
\hline (6) & There are arm supports on the chair? (yes/no) ${ }^{\dagger}$ & $13 / 7$ & $16 / 4$ & 0.29 \\
\hline (7) & The back of the chair is supportive? (yes/no) ${ }^{\dagger}$ & $16 / 4$ & $16 / 4$ & 1.0 \\
\hline (8) & The back of the chair is adjustable? (yes/no) ${ }^{\dagger}$ & $6 / 14$ & $5 / 15$ & 0.72 \\
\hline (9) & There is a lumbar support? (yes/no)* & $4 / 16$ & $4 / 16$ & 1.0 \\
\hline$(10)$ & Elbow angles are $70-90$ degrees? (yes/no)* & $17 / 3$ & $16 / 4$ & 1.0 \\
\hline$(11)$ & Elbows are supported when using a keyboard? (yes/no) ${ }^{\dagger}$ & $8 / 12$ & $10 / 10$ & 0.53 \\
\hline (12) & Elbows are supported when using a mouse? (yes/no) ${ }^{\dagger}$ & $13 / 7$ & $13 / 7$ & 1.0 \\
\hline (13) & $\begin{array}{l}\text { Wrist angle is within } 10 \text { degrees of neutral when using a keyboard? } \\
\text { (yes/no)* }\end{array}$ & $4 / 16$ & $5 / 15$ & 1.0 \\
\hline (14) & The feet touch on the floor? (yes/no)* & $19 / 1$ & $20 / 0$ & 1.0 \\
\hline$(15)$ & $\begin{array}{l}\text { The angle of the knees is } 70 \text { to } 90 \text { degrees and buttock's angle is } 90 \\
\text { degree? (yes/no) }\end{array}$ & $5 / 15$ & $8 / 12$ & 0.31 \\
\hline$(16)$ & There is a copy holder on the monitor? (yes/no)* & $2 / 18$ & $5 / 15$ & 0.41 \\
\hline (17) & The neck is in a neutral position when looking at the monitor? (yes/no) ${ }^{\dagger}$ & $13 / 7$ & $14 / 6$ & 0.74 \\
\hline
\end{tabular}

*The Fisher's exact test was used for statistical analysis. ${ }^{\dagger}$ The Chi-squared test was used for statistical analysis.

MYMOP2 score significantly decreased in the cupping group compared with the heating pad group. The eta-squared values suggested that cupping had a large effect in improving symptoms related to VDT work at 3 weeks $(0.1425)$ and at 7 weeks $(0.1601$, Table 3$)$.

\section{NDI and ROM of C-spine}

The mean NDI score also decreased in the cupping group at 3 and 7 weeks, respectively, compared with the heating pad group. The effect sizes were very large at 3 weeks (0.1509) and 7 weeks $(0.3136$, Table 3). The analysis of individual section scores using ANCOVA suggested that cupping treatment showed significant improvement in reading $(p=0.039)$, headaches $(p=0.001)$, work $(p=0.002)$ and sleeping $(p=0.033)$ after 2 weeks of treatment when compared with heating pad application. In addition, significant differences were observed in all the sections except reading at 7 weeks. Among the cervical ROM changes, significant improvement was observed only in neck extension at 7 weeks (ANCOVA, $p=0.0491$, Fig. 3).

\section{$E Q-5 D$ index}

The median EQ-5D index did not show a significant difference between the 2 groups at 3 weeks (0.91 [0.91, 1.0] in the cupping group and heating pad group), but a significant difference in favor of cupping treatment was observed at 7 weeks. Effect sizes showed the same pattern; a small to medium effect was reported at 3 weeks $(0.0427)$ but a large effect was observed at 7 weeks $(0.1537$, Table 3$)$.

\section{Adverse events}

Four participants complained of adverse events related to the cupping therapy: skin laceration, whole body itching, pain at the cupping sites and generalized body ache. Except for the whole body itching, the other 3 symptoms were assumed to be caused by the cupping therapy. All of the symptoms were mild and disappeared within several days.

\section{Discussion}

Originally, this was a preliminary study with a minimal sample size to lay the groundwork for a future trial in which we would evaluate the effect of cupping therapy for neck pain in VDT users. However, when we evaluated the ideal sample size for testing the primary outcome difference (NRS) between the cupping and heating pad groups, we found that for a result with a 0.05 for $\alpha, 0.2$ for $\beta$ and a $10 \%$ for drop-out rate, only 15 participants for each group were required. Cupping was considerably effective in improving pain and function of the neck and relieving bothersome symptoms of VDT users in this study. Quality of life also largely improved after treatment. The body's response to stress and fatigue, 
(A) First treatment

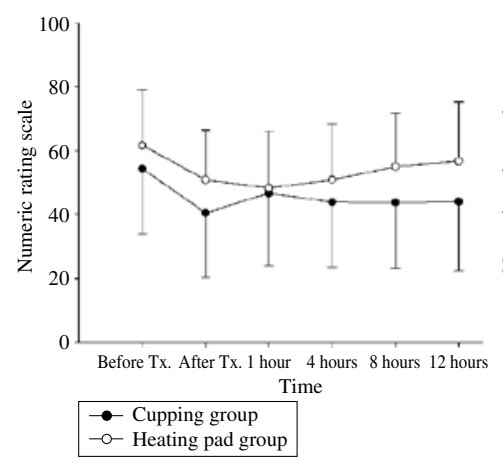

(D) Fourth treatment

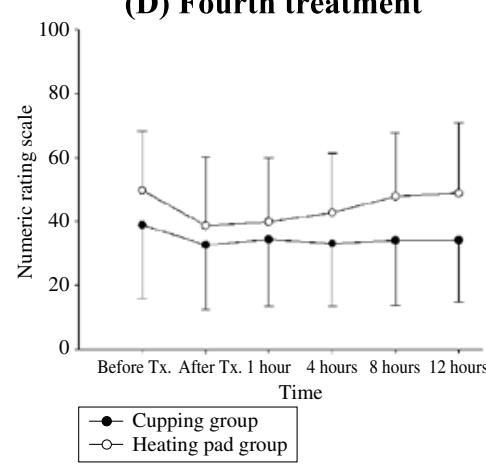

(B) Second treatment

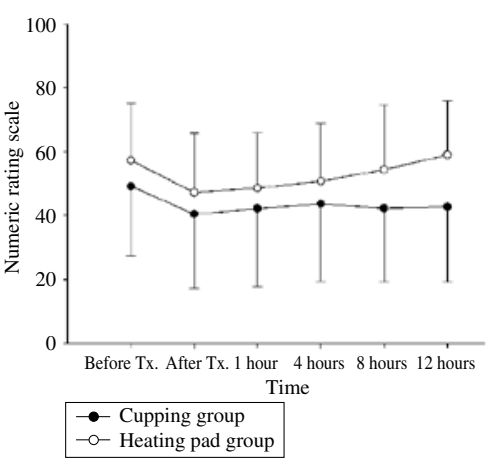

(E) Fifth treatment

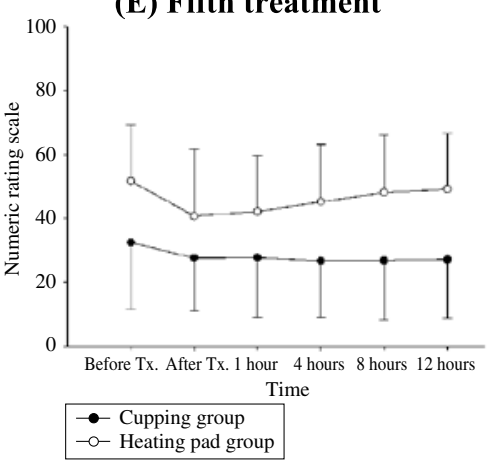

(C) Third treatment

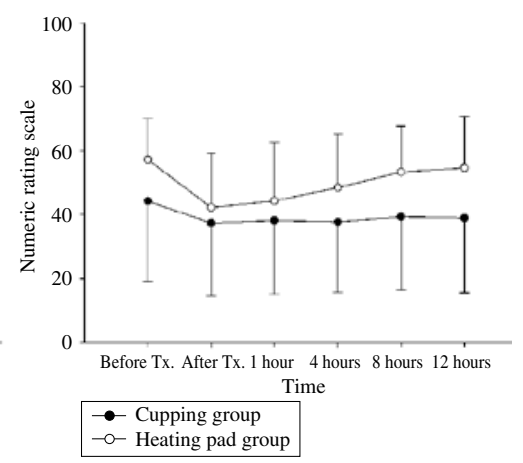

(F) Sixth treatment

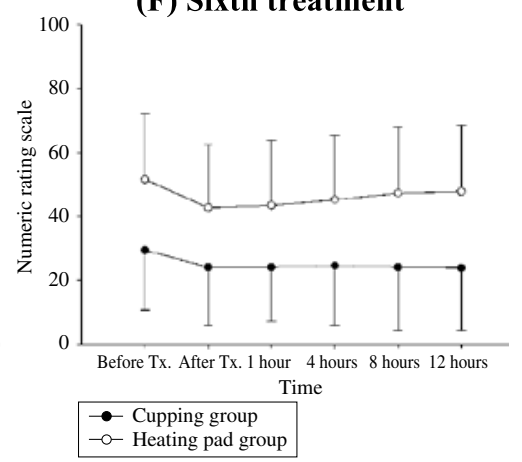

Fig. 2. The time series numeric rating scale data on neck pain before and after treatment.

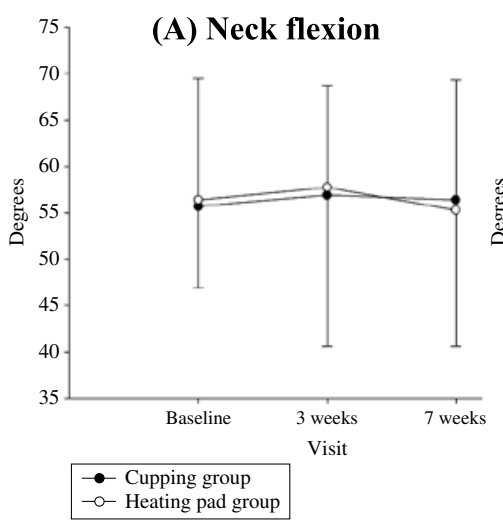

(D) Neck Rt. lateral flexion

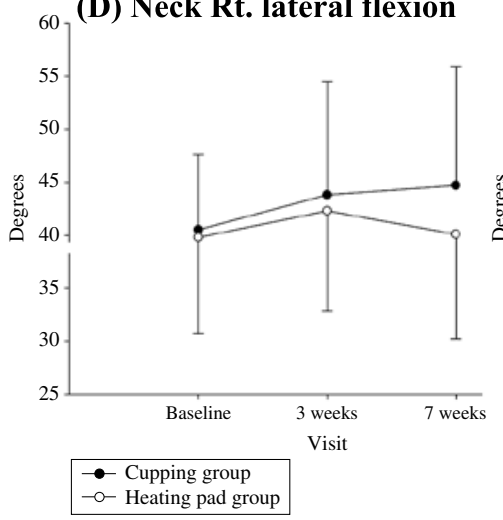

(B) Neck extension

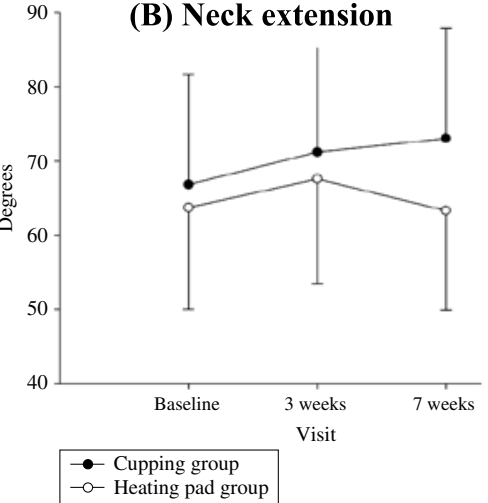

(E) Neck Lt. rotation

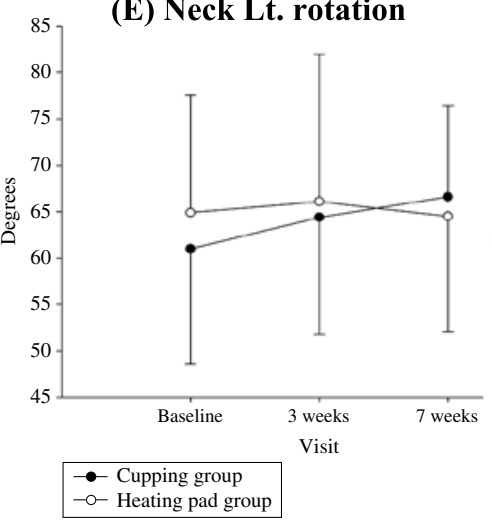

(C) Neck Lt. lateral flexion

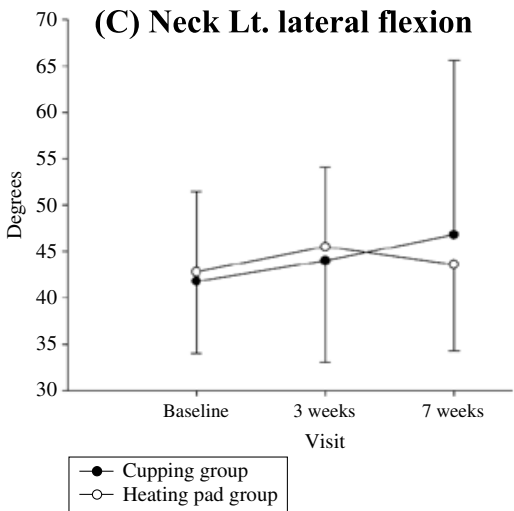

(F) Neck Rt. rotation

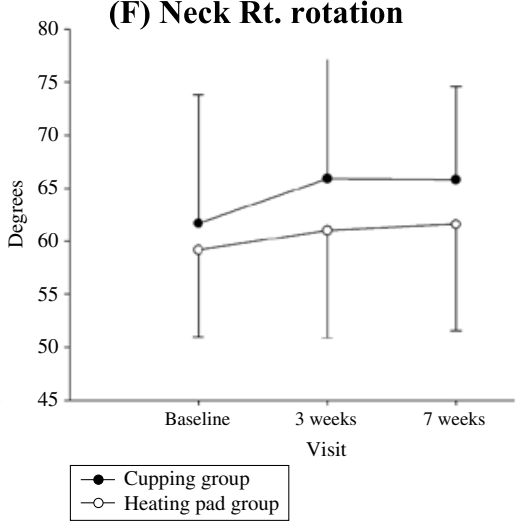

Fig. 3. The change in cervical spine range of motion. 
Table 3. Primary and secondary outcomes at each visit

\begin{tabular}{|c|c|c|c|c|c|c|c|c|c|c|}
\hline & \multicolumn{3}{|c|}{$\begin{array}{l}\text { Cupping group } \\
(\mathrm{n}=20, \text { mean, } S D)\end{array}$} & \multicolumn{3}{|c|}{$\begin{array}{l}\text { Heating pad group } \\
(\mathrm{n}=20, \text { mean, } S D)\end{array}$} & \multicolumn{2}{|c|}{$p$-value } & \multicolumn{2}{|c|}{ Effect size $\left(\eta^{2}\right)$} \\
\hline & Baseline & 3 weeks & 7 weeks & Baseline & 3 weeks & 7 weeks & 3 weeks & 7 weeks & 3 weeks & 7 weeks \\
\hline $\begin{array}{l}\text { NRS } \\
\text { (mean, SD) }\end{array}$ & $\begin{array}{l}59.25 \\
16.33\end{array}$ & $\begin{array}{l}28.55 \\
17.83\end{array}$ & $\begin{array}{l}28.75 \\
21.87\end{array}$ & $\begin{array}{l}64.85 \\
14.89\end{array}$ & $\begin{array}{l}48.3 \\
18.16\end{array}$ & $\begin{array}{l}50.3 \\
21.26\end{array}$ & $0.025^{*}$ & $0.005^{*}$ & 0.1642 & 0.1262 \\
\hline $\begin{array}{l}\text { MYMOP2 } \\
\text { (mean, SD) }\end{array}$ & $\begin{array}{l}3.43 \\
0.88\end{array}$ & $\begin{array}{l}2.27 \\
1.08\end{array}$ & $\begin{array}{l}2.03 \\
0.96\end{array}$ & $\begin{array}{l}3.56 \\
0.83\end{array}$ & $\begin{array}{l}3.09 \\
0.86\end{array}$ & $\begin{array}{l}3.03 \\
1.15\end{array}$ & $0.0127 *$ & $0.0035 *$ & 0.1425 & 0.1601 \\
\hline $\begin{array}{l}\text { NDI score, } \% \text { point } \\
\text { (mean, SD) }\end{array}$ & $\begin{array}{l}23.33 \\
10.41\end{array}$ & $\begin{array}{l}11.57 \\
8.17\end{array}$ & $\begin{array}{l}10.19 \\
5.99\end{array}$ & $\begin{array}{l}22.96 \\
8.61\end{array}$ & $\begin{array}{l}19.26 \\
10.95\end{array}$ & $\begin{array}{l}20.63 \\
9.82\end{array}$ & $0.0039 *$ & $<0.0001^{*}$ & 0.1509 & 0.3136 \\
\hline $\begin{array}{l}\text { EQ-5D index } \\
\text { (median [25\% to } \\
75 \% \mathrm{IQR}] \text { ) }\end{array}$ & $\begin{array}{l}0.91 \\
{[0.87} \\
0.91]\end{array}$ & $\begin{array}{l}0.91 \\
{[0.91} \\
1.0]\end{array}$ & $\begin{array}{l}1.0 \\
{[0.88,} \\
1.0]\end{array}$ & $\begin{array}{l}0.89 \\
{[0.86} \\
0.91]\end{array}$ & $\begin{array}{l}0.91 \\
{[0.86} \\
0.91]\end{array}$ & $\begin{array}{l}0.91 \\
{[0.86} \\
0.91]\end{array}$ & 0.1072 & $0.0054 *$ & 0.0427 & 0.1537 \\
\hline SRI-SF & $\begin{array}{l}36.5 \\
15.87\end{array}$ & $\begin{array}{l}32 \\
10.08\end{array}$ & $\begin{array}{l}33.35 \\
11.95\end{array}$ & $\begin{array}{l}40.1 \\
12.68\end{array}$ & $\begin{array}{l}35.8 \\
9.51\end{array}$ & $\begin{array}{l}37.85 \\
8.95\end{array}$ & 0.3643 & 0.2921 & 0.0114 & 0.0180 \\
\hline FSS & $\begin{array}{l}3.10 \\
1.29\end{array}$ & $\begin{array}{l}2.61 \\
1.23\end{array}$ & $\begin{array}{l}2.33 \\
1.09\end{array}$ & $\begin{array}{l}3.31 \\
1.41\end{array}$ & $\begin{array}{l}3.04 \\
1.16\end{array}$ & $\begin{array}{l}3.02 \\
1.35\end{array}$ & 0.1980 & 0.0511 & 0.0140 & 0.0480 \\
\hline
\end{tabular}

ANCOVA was used for the statistical analysis of changes from baseline between the two groups with regard to each outcome at the primary and secondary end points (covariate: baseline value). ${ }^{*} p<0.05$. NRS: numeric rating scale, MYMOP2: Measure yourself medical outcome profile 2, NDI: neck disability index, IQR: interquartile range, SRI-SF: short form of stress response inventory, FSS: fatigue severity scale.

which may be affected by VDT work, was improved by both tested treatments; however, there were no significant differences between the two groups. When compared with heating pad therapy, cupping increased neck-extension significantly, but there were no other significant differences in C-spine ROM. Four participants in the cupping group reported mild adverse events related to the treatment. Because the participants had concomitant disturbing symptoms along with neck pain that might affect the outcome assessment of this study, the results need to be interpreted carefully. However, the results of this study provide good evidence that cupping may be a helpful, when combined with self-care, non-drug intervention for VDT-worker neck pain.

The methodologically low quality of past clinical trials on cupping has been noted as a big obstacle for conclusive evidence of the effectiveness of cupping therapy. Domains such as allocation concealment, incomplete outcome data and selective outcome reporting were not appropriately or never reported in most of the studies ${ }^{29}$. In addition, most of the cupping studies adopted cupping not as a single intervention but in combination with acupuncture or other treatment modalities from which we cannot estimate the specific effect of cupping therapy ${ }^{29)}$. We conducted this study with methodological rigor to eliminate serious bias and compared the effect of cupping for neck pain to heating pad therapy without other co-interventions. Thus, these results suggest the overall effec- tiveness of cupping itself.

Recent studies on the effectiveness of cupping for neck pain suggest a short-term effect of cupping during or after the treatment ${ }^{30,31)}$. In our study, we evaluated the short-term effect and mid-term effect following treatment as well. Cupping demonstrated immediate effects on neck pain that were maintained for at least 12 hours after treatment, and the therapeutic effects on the pain and function of neck were maintained with comparatively large effect sizes until 1 month after the end of the treatment. Similar results were not demonstrated in the heating pad group.

In this study, we only selected VDT workers with chronic and moderate-to-severe neck pain. In accordance with the wide usage of computers and smartphones in modern society, neck pain related to VDT work has increased sharply and affects everyday life ${ }^{6}$. Together with modifications of life style and workstations, an appropriate treatment for neck pain is needed to improve work efficiency and public health. Various complementary and alternative (CAM) therapies have been suggested to have immediate or short-term effects on neck pain ${ }^{32}$, but few studies have evaluated the effect in VDT workers. In this sense, it is meaningful and useful in a modern society to evaluate the problems related to VDT work as we did in this study.

There are several limitations in this study. First, this was an open trial where participants and research- 
ers knew the allocation results during the study. The participants of both groups had comparatively high expectations on the effectiveness of cupping for relieving neck pain. The average expectation score was 7 points out of 10 (high expectation) in both groups (Table 1). Patient expectation concerning the effectiveness of specific interventions affects the health-related outcomes $^{33)}$, so blinding of assigned interventions is a critical factor for avoiding the introduction of serious bias in randomized controlled trials. However, even though a sham cupping device has been developed recently ${ }^{34)}$, it could not be used in this study because that device was validated only for application to low-back lesions, and a sham cupping devise attached by adhesives is easily unmasked by participants according to a previous report ${ }^{31}$. Due to these factors, we did not adopt a sham or placebo control. As a result, there exists a high risk of bias attributable to the non-blinded nature of this study that should be considered when interpreting the observed favorable results in this study. However, we attempted to maintain rigor in two other important domains including allocation concealment and outcome assessor blinding, which may significantly exaggerate the true effects of intervention when performed poorly ${ }^{35,36)}$. Second, to ensure reproducibility of the study results and to improve the quality of clinical practice, the cupping therapy was standardized appropriately and described in detail. This was a pragmatically designed study in which the methods for cupping were decided within minimal guidelines by the individual practitioner. We allowed freedom for the selection of points and stimulation methods (wet or dry cupping) to reflect the real-world clinical situation. As it is a very difficult to separate individual factors affecting the specific effects of cupping, most clinical research does not adopt strict standardized treatment guidelines for the cupping procedure, including the size of cups, applied areas, volume of drained air, and retention time of

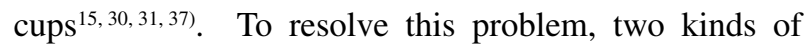
efforts should be made in the future. First, experimental research needs to be conducted to identify the separate physical properties of cupping and evaluate the degrees of their contributions to the observed effects. The other is the establishment of specific reporting guidelines for cupping trials similar to standards for other CAM treatment modalities, such as the STandards for Reporting Interventions in Clinical Trials of Acupuncture (STRICTA) ${ }^{38}$.

Given the observed clinical effectiveness of cupping for neck pain, we still need to elucidate possible therapeutic mechanisms. Contrary to evidence that suggests the effectiveness of cupping relates to the various conditions of the clinical trials ${ }^{29}$, there are a few studies that suggest a basic mechanism. Several studies have suggested that wet cupping can improve chronic inflammation through an immunomodulatory effect $^{39,40)}$. Another study suggested that cupping may induce hematological changes (i.e., lowering serum lipid concentrations $)^{41)}$. More likely possibilities include the activation of diffuse noxious inhibitory control (DNIC) triggered by tactile stimulus and negative pressure ${ }^{42)}$ and the recovery of chronic soft tissues injuries resulting from improved local blood circulation $^{31)}$. These two mechanisms may contribute to pain relief and functional improvement in the neck as a specific effect of cupping. These hypotheses should be examined in future studies.

Additionally, there is one additional aspect that needs to be discussed. Environmental factors related to VDT work were observed with every participant, and several factors did not meet the standard guidelines for workstations. Inappropriate postures contribute to musculoskeletal symptoms for office workers ${ }^{2)}$ and office furniture, such as chairs, computer tables, display monitors, keyboards and mice, may affect bad sitting posture. A modification of such work environments and health education for office workers appears necessary to prevent VDT work-related symptoms.

In conclusion, the results of this pragmatic study suggest that 2 weeks of cupping therapy with an exercise program may be effective in reducing pain and improving neck function in VDT workers. Future studies testing the efficacy of cupping and using an appropriate sham device will be helpful in evaluating the specific effects of cupping.

Acknowledgments: This study was supported by the "Development of Acupuncture, Moxibustion and Meridian Standard Health Technology" project of the Korea Institute of Oriental Medicine (K12010). We especially appreciate to Sun-Mi Choi, who allowed us to conduct this clinical trial as a "Development of Acupuncture, Moxibustion and Meridian Standard Health Technology" project.

\section{References}

1) Iwakiri K, Mori I, Sotoyama M, et al. Survey on visual and musculoskeletal symptoms in VDT workers. Sangyo Eiseigaku Zasshi 2004; 46: 201-12 (in Japanese).

2) Eltayeb S, Staal JB, Hassan A, de Bie RA. Work related risk factors for neck, shoulder and arms complaints: a cohort study among Dutch computer office workers. J Occup Rehabil 2009; 19: 315-22.

3) Janwantanakul P, Pensri P, Jiamjarasrangsri V, Sinsongsook T. Prevalence of self-reported musculoskeletal symptoms among office workers. Occup Med (Lond) 2008; 58: 436-8.

4) Uchino $M$, Schaumberg DA, Dogru $M$, et al. Prevalence of dry eye disease among Japanese visu- 
al display terminal users. Ophthalmology 2008; 115 : 1982-8.

5) Taino G, Ferrari M, Mestad IJ, Fabris F, Imbriani M. Asthenopia and work at video display terminals: study of 191 workers exposed to the risk by administration of a standardized questionnaire and ophthalmologic evaluation. G Ital Med Lav Ergon 2006; 28: 487-97.

6) Waersted M, Hanvold TN, Veiersted KB. Computer work and musculoskeletal disorders of the neck and upper extremity: a systematic review. BMC Musculoskelet Disord 2010; 11: 79.

7) Park HS, Lee YK, Ok DM. Comparison of ministry of labor notification for VDT work management and KS A ISO 9241. Journal of the Ergonomics Society of Korea 2009; 28: 109-15.

8) Farhadi K, Schwebel DC, Saeb M, Choubsaz M, Mohammadi R, Ahmadi A. The effectiveness of wet-cupping for nonspecific low back pain in Iran: a randomized controlled trial. Complement Ther Med 2009; 17: 9-15.

9) Michalsen A, Bock S, Ludtke R, et al. Effects of traditional cupping therapy in patients with carpal tunnel syndrome: a randomized controlled trial. J Pain 2009; 10: 601-8.

10) Han CH, Kim SW, Lee Sd, Shin MS, Shin Sh, Choi SM. Telephone survey for grasping clinical actual state of blood letting therapeutics in Korea. The Journal of Korean Acupuncture and Moxibustion Society 2006; 23: 177-87.

11) Han CH, Kim SW, Shin MS, Choi SM. An interview survey for grasping clinical actual state of blood letting therapeutics in korea. The Journal of Korean Acupuncture and Moxibustion Society 2007; 24: 9-18.

12) Guidance document for classification and class of medical devices. [Online]. 2010 [cited 2012 Mar 20]; Available from: URL: http://www.law.go.kr/admRulLsInfoP.do?admRulSeq=2000000016930\#AJAX

13) Lee BY, Song YK, Lim HH. Literature investigation regarding cupping therapy and analysis of current professional's cupping treatment. J Oriental Rehab Med 2008; 18: 169-91 (in Korean).

14) Fu LM, Li JT, Wu WS. Randomized controlled trials of acupuncture for neck pain: systematic review and meta-analysis. J Altern Complement Med 2009; 15: 133-45.

15) Kim JI, Kim TH, Lee MS, et al. Evaluation of wet-cupping therapy for persistent non-specific low back pain: a randomised, waiting-list controlled, open-label, parallel-group pilot trial. Trials 2011; 12: 146.

16) Shao M. Clinical observations on the treatment of 93 cervical spondylopathy by dazhui blood-letting puncturing and cupping. Shanghai J Acu-mox 2003; 12: $20-1$.

17) Zhao M. Treating disease of vertebrae cervicales in neck-type with bamboo jar. Journal of Tianjin University of Traditional Chinese Medicine 2010; 29: $20-1$.
18) Garra G, Singer AJ, Leno R, et al. Heat or cold packs for neck and back strain: a randomized controlled trial of efficacy. Acad Emerg Med 2010; 17: 484-9.

19) Shin D, Shin W, Song C. The effects of a deep cervical flexion exercise for sedentary workers with VDT syndrome. Journal of the Ergonomics Society of Korea 2008; 27: 59-64.

20) Gwendolen J, Michele S, Deborah F, Julia T, Shaun OL. Chapter 14 therapeutic exercise for cervical disorders: practice pointers whiplash, headache, and neck pain: Churchill Livingstone 2008.

21) Paterson C. Complementary practitioners as part of the primary health care team: consulting patterns, patient characteristics and patient outcomes. Fam Pract 1997; 14: 347-54.

22) Song KJ, Choi BW, Kim SJ, Yoon SJ. Cross-cultural adaptation and validation of the Korean version of the neck disability index. J Korean Orthop Assoc 2009; 44: 350-9.

23) Nam HS, Kim GY, Kwon SS, et al. EQ-5D Korean valuation study using time trade off method, Korea Centers for Disease Control \& Prevention 2007.

24) Choi SM, Kang TY, Woo JM. Development and validation of a modified form of the stress response inventory for workers. J Korean Neuropsychiatr Assoc 2006; 45: 541-53 (in Korean).

25) Chung KI, Song CH. Clinical usefulness of fatigue severity scale for patients with fatigue, and anxiety or depression. Korean J Psychosomatic Medicine 2001; 9: 164-73.

26) WHO toxicity grading scale for determining the severity of adverse events. [Online]. 2003 [cited 2011 May 13]; Available from: URL: http://www. icssc.org/Documents/Resources/AEManual2003Appe ndicesFebruary_06_2003\%20final.pdf

27) Julious SA. Sample size of 12 per group rule of thumb for a pilot study. Pharmaceutical Statistics 2005; 4: 287-91.

28) Ellis PD. The Essential Guide to Effect Sizes. Cambridge (U.K.): Cambridge Univeristy Press; 2010.

29) Cao H, Li X, Liu J. An updated review of the efficacy of cupping therapy. PLoS One 2012; 7: e31793.

30) Cramer H, Lauche R, Hohmann C, et al. Randomized controlled trial of pulsating cupping (pneumatic pulsation therapy) for chronic neck pain. Forsch Komplementmed 2011; 18: 327-34.

31) Lauche R, Cramer H, Choi KE, et al. The influence of a series of five dry cupping treatments on pain and mechanical thresholds in patients with chronic non-specific neck pain-a randomised controlled pilot study. BMC Complement Altern Med 2011; 11: 63.

32) Furlan AD, Yazdi F, Tsertsvadze A, et al. A systematic review and meta-analysis of efficacy, cost-effectiveness, and safety of selected complementary and alternative medicine for neck and low-back pain. Evid Based Complement Alternat Med 2012; 2012: 953139. 
33) Mondloch MV, Cole DC, Frank JW. Does how you do depend on how you think you'll do? A systematic review of the evidence for a relation between patients' recovery expectations and health outcomes. CMAJ 2001; 165: 174-9.

34) Lee MS, Kim JI, Kong JC, Lee DH, Shin BC. Developing and validating a sham cupping device. Acupunct Med 2010; 28: 200-4.

35) Schulz KF, Grimes DA. Allocation concealment in randomised trials: defending against deciphering. Lancet 2002; 359: 614-8.

36) Hrobjartsson A, Thomsen AS, Emanuelsson F, et al. Observer bias in randomised clinical trials with binary outcomes: systematic review of trials with both blinded and non-blinded outcome assessors. BMJ 2012; 344: e1119.

37) Lauche R, Cramer H, Hohmann C, et al. The effect of traditional cupping on pain and mechanical thresholds in patients with chronic nonspecific neck pain: a randomised controlled pilot study. Evid Based Complement Alternat Med 2012; 2012:
429718.

38) MacPherson H, Altman DG, Hammerschlag R, et al. Revised STandards for Reporting Interventions in Clinical Trials of Acupuncture (STRICTA): extending the CONSORT statement. Acupunct Med 2010; 28: 83-93.

39) Ahmed SM, Madbouly NH, Maklad SS, Abu-Shady EA. Immunomodulatory effects of blood letting cupping therapy in patients with rheumatoid arthritis. Egypt J Immunol 2005; 12: 39-51.

40) Liu Y, Zeng Z, Liang F, Fu Y, Chen J, Li X. Effects of bloodletting therapy on HSP70 and IL-1 in adjuvant arthritic rat local diseased area. Medical Acupuncture 2005; 16: 30-2.

41) Niasari M, Kosari F, Ahmadi A. The effect of wet cupping on serum lipid concentrations of clinically healthy young men: a randomized controlled trial. J Altern Complement Med 2007; 13: 79-82.

42) Calvino B, Grilo RM. Central pain control. Joint Bone Spine 2006; 73: 10-6.

\section{Appendix}

(A)



(B)

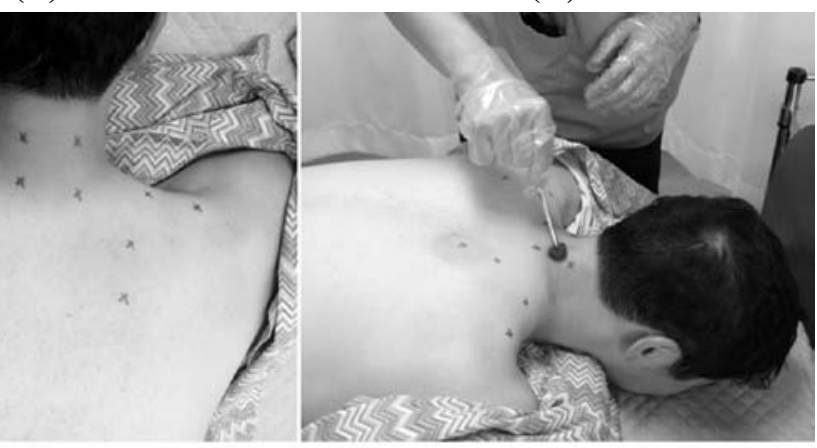

(D)

(E)

(F)

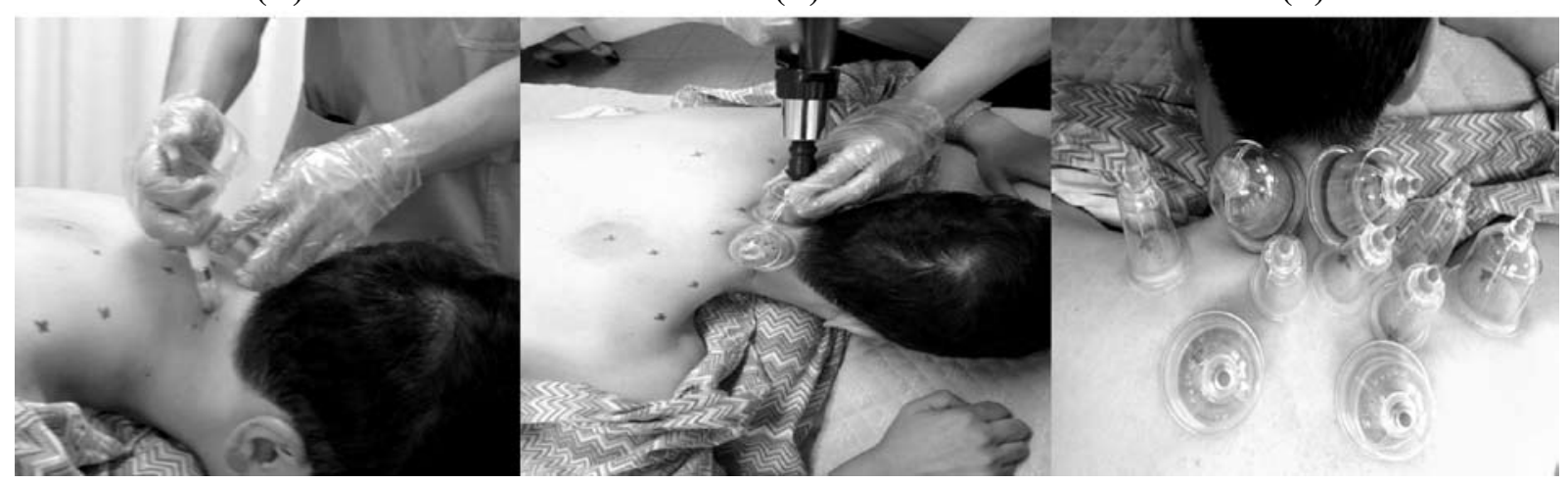

The procedure of the cupping treatment.

(A) Preparation

(B) Finding points

(C) Swabbing of the points

(D) Puncturing 1 to 2 points

(E) Pumping and exhausting inner air from the cups

(F) Retaining the cups 